\title{
Digital Therapeutics in Parkinson's Disease: Practical Applications and Future Potential
}

\author{
Terry D. Ellis ${ }^{\mathrm{a}, *}$ and Gammon M. Earhart ${ }^{\mathrm{b}}$ \\ ${ }^{a}$ Department of Physical Therapy \& Athletic Training, Center for Neurorehabilitation, College of Health and \\ Rehabilitation Sciences: Sargent College, Boston University, Boston, MA, USA \\ ${ }^{\mathrm{b}}$ Program in Physical Therapy, Department of Neurology, Department of Neuroscience, \\ Washington University in St. Louis School of Medicine, St. Louis, MO, USA
}

Accepted: 31 January 2021

Pre-press 23 February 2021

\begin{abstract}
Digital therapeutics, treatments delivered remotely and enabled by modern technology, facilitate the provision of personalized, evidence-based, interdisciplinary interventions to manage the complexities associated with Parkinson's disease. In the context of the COVID-19 pandemic, the need for digital therapeutics has arguably never been greater. However, despite new advances in technology and a heightened interest due to the pandemic, digital therapeutics remain underdeveloped and underutilized. In this paper, we briefly review practical applications and emerging advances in digital therapeutic platforms that target motor and non-motor signs and healthy lifestyle behaviors such as regular exercise, a healthful diet and optimal sleep hygiene habits. Future applications which could transform personalized self-management and patient care are presented. Opportunities, drawbacks and barriers to access are discussed.
\end{abstract}

Keywords: Parkinson's disease, digital therapeutics, virtual coach, mobile health

\section{INTRODUCTION}

Parkinson's disease (PD) is a multifaceted condition that may include a host of motor and non-motor symptoms. Given the complexities of the condition, it is widely recognized that an integrated, interdisciplinary approach to treatment is warranted to live optimally with PD [1]. However, despite evidence regarding the beneficial effects of many therapeutic approaches, access to these approaches is restricted for many reasons including lack of transportation, limited numbers of trained specialists in one's vicinity and, more recently, the COVID-19 pandemic.

${ }^{*}$ Correspondence to: Terry D. Ellis, PT, PhD, FAPTA, Department of Physical Therapy \& Athletic Training; Center for Neurorehabilitation, College of Health and Rehabilitation Sciences: Sargent College, Boston University; 635 Commonwealth Avenue, Boston, MA 02215, USA. Tel.: +1 617353 7571; E-mail: tellis@bu.edu.
Furthermore, treatment administered by healthcare professionals is typically provided in a clinical setting at a discrete point in time when the symptoms or challenges faced by patients may not be manifesting. Digital therapeutics offer a scalable mechanism to overcome the barriers and shortcomings associated with traditional in-clinic care by delivering personalized evidence-based treatment remotely at a time and place most convenient and beneficial to the patient. Digital therapeutics are distinct from other digital health approaches in that their primary function is to deliver software-generated therapeutic interventions directly to patients to prevent, manage or treat a medical condition [2,3]. Approaches may be used independently or in conjunction with cliniciandelivered treatment. Digital therapeutics can also be integrated with mobile health platforms and may be paired with devices, sensors or wearables, allowing more robust functionalities [3]. The need for digital 
therapeutics has arguably never been greater, and these approaches are currently underdeveloped and underutilized [4]. This paper focuses on recent and emerging evidence for the use of digital therapeutics to facilitate lifestyle changes and to address both motor (e.g., walking) and non-motor (e.g., sleep, anxiety) aspects of PD with the goal of improving patient outcomes.

\section{ARTIFICIALLY INTELLIGENT VIRTUAL COACHES TO PROMOTE HEALTHY LIFESTYLE BEHAVIORS}

Advances in digital therapeutics provide numerous options to foster engagement in healthy lifestyle behaviors such as regular exercise, a healthful diet and optimal sleep hygiene habits. Despite the known benefits of these healthy lifestyle practices, making and sustaining lifestyle modifications is tremendously challenging, particularly in the context of motor and non-motor signs associated with PD. Many approaches using digital therapeutics encompass strategies, grounded in cognitive behavioral change theory, known to be effective in fostering changes in behavior [5]. These strategies may consist of goal setting, action planning, self-monitoring as well as the provision of social support, positive feedback and rewards. All of these aspects can be addressed through use of relational agents which are computational artifacts designed to establish long-term social-emotional relationships with users, fostering a therapeutic alliance in an automated fashion. In an early study in PD, a relational agent took the form of an animated virtual coach that had face-to-face conversions with users who "talked" to the character through a touch screen on a tablet computer in their homes [6]. Five-minute daily counseling sessions over one month were designed to establish a social bond with users and improve motivation and self-efficacy through employing behavioral change strategies with the goal of increasing daily walking. Participants interacted with the virtual exercise coach 25.4 days out of the recommended 30 days, mean adherence to daily walking was $85 \%$ and gait speed and walking capacity improved. However, this form of a virtual coach only allowed users to respond to agent-directed questions which encompassed a fixed set of multiple choice responses and users were not able to ask questions.

In contrast, more recent developments in virtual coach technology allow recognition of free-written or spoken language (natural language processing), enabling user-directed, personalized communication with more humanistic qualities [7]. Advances in machine learning, particularly in neural networks, have allowed for more complex dialogue management methods and conversational flexibility [7]. Applications to healthcare, particularly behavioral change interventions, are emerging. In a 12-week single-arm pre-post study focused on increasing physical activity and fostering adoption of a Mediterranean-style diet in inactive community-dwelling adults aged 45-75 $(\mathrm{N}=81)$, an artificially intelligent virtual coach was deployed on a cloud-based instant messaging platform and downloaded on users' personal devices [8]. The virtual coach guided participants through an introductory educational session, 11 weekly checkins regarding daily step counts and dietary patterns, assisted users with setting personalized step and diet goals and was available 24/7 to answer questions. Participants increased physical activity by a mean of 109.8 minutes per week and experienced significant weight loss over 12 weeks. Feasibility was excellent in terms of recruitment, retention ( $90 \%$ at 12 weeks) and safety [8]. Limitations of this study include a lack of a control condition and a relatively short followup period. Well controlled trials in PD are needed to determine the effectiveness of this approach over the long-term. With advances in artificial intelligence (AI), virtual coaches have tremendous potential to promote self-management of the condition across multiple domains (e.g., physical activity, diet, sleep).

\section{DIGITAL THERAPEUTICS TO IMPROVE WALKING OUTCOMES}

A digital therapeutic platform (MedRhythms Inc.) has been designed to improve walking outcomes by digitizing a well-established, evidence-based intervention known as Rhythmic Auditory Stimulation (RAS). RAS is an approached used in rehabilitation in which external auditory cues (e.g., beat of a metronome) are applied to facilitate entrainment or the ability to synchronize movements (i.e., walking) to a beat [9]. Several studies reveal the beneficial effects of RAS in improving spatiotemporal parameters of gait in PD [10], but translation of this approach into digital therapeutics is just beginning. The MedRhythms digital therapeutic platform is a closed loop system that (see Fig. 1) consists of an inertial sensor that monitors a patient's real time walking cadence, a Bluetooth low-energy radio chip 


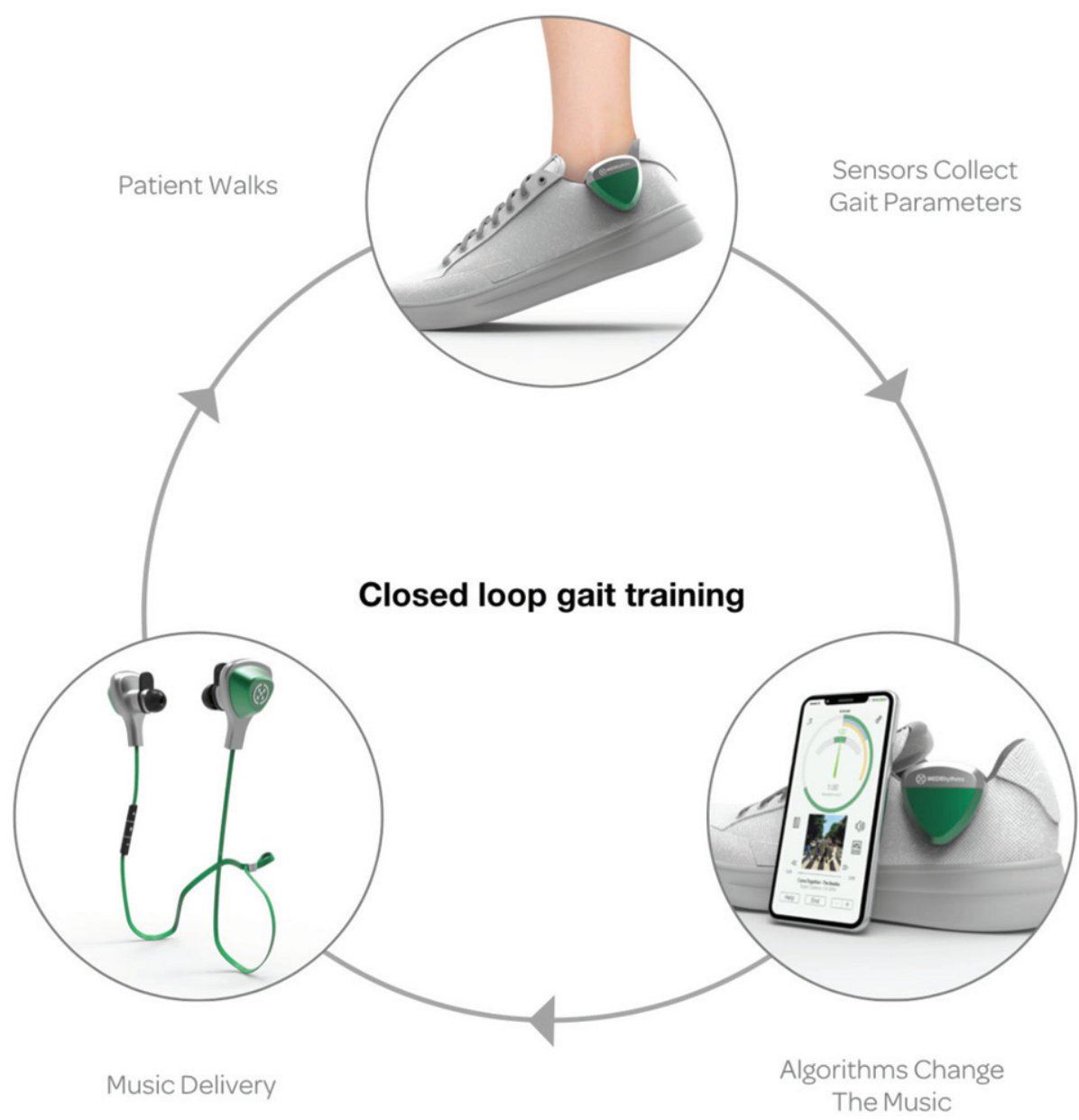

Fig. 1. Digital Closed Loop Gait Training Therapeutic In the top portion of the figure, a sensor is worn on the shoe to collect real-time walking cadence. A smartphone (lower right) houses the music playlist and delivers the acoustic signals through headphones worn (lower left) by the patient. Algorithms time shift the music to match the patient's cadence and modulate according to entrainment outcomes.

with near field communication and a floating-pointon-board processor. A smartphone houses the music playlist and delivers the acoustic signals through bone-conducting headphones. Automatic decision processing occurs that adapts auditory stimuli based on preferred cadence as well as their synchronization performance. As synchronization improves, tempo of the music is increased to further challenge walking. This approach was recently applied to persons with stroke in a proof-of-concept study [11]. Results revealed that a single, fully automated training visit resulted in significant increases in comfortable and fast walking speeds. No trips or falls occurred during the training. All users reported the device helped them walk faster and $70 \%$ indicated they would use it most or all of the time at home [11]. Though these results are limited to one session of training, this approach has much potential for application in PD and could allow access to a home-based approach to gait training to enhance quality and quantity of walking and extend the benefits of in-person training with a physical therapist.

Digital therapeutics can also be integrated with mobile health applications ("apps"). Mobile health (mHealth) offers a remote platform to increase access to physiotherapists (and other healthcare professionals) with expertise in PD. The delivery enhancement of lifestyle interventions through mHealth "apps" provides a mechanism to provide ongoing, rather than episodic, goal setting; provision of real-time, rather than delayed, feedback to reinforce positive behavioral change; more frequent adjustments of individually tailored programs; and ongoing connections with healthcare professionals through messaging features $[12,13]$. Digital therapeutics provided through mobile health apps allow physiotherapy interventions to 
be more readily scaled, providing access to greater numbers of people who may not otherwise have access. These approaches can be seamlessly integrated into everyday life thereby allowing greater flexibility, adaptability, and relevance to the individual. In a single-blinded comparative effectiveness RCT, changes in walking activity were compared in an mHealth-mediated exercise program and an active control over one year in persons with mild to moderate PD [14]. At an initial clinic visit, participants were instructed in individually tailored exercises which were video-recorded and uploaded to the app (Wellpepper, Inc.) on a tablet. Participants accessed the exercises at home via the mobile app and entered data on adherence, pain and level of difficulty which was monitored remotely by the physiotherapist. This store-and-forward approach allowed data to be collected and viewed asynchronously by a physiotherapist remotely. Participants could also message the physiotherapist with questions, allowing troubleshooting and motivational encouragement. Exercises were progressed and adapted remotely by the physiotherapist based on the data reviewed. Increases in daily steps and moderate intensity minutes were observed in both groups over one year; however, changes were clinically meaningful only in the less active subgroup receiving the mHealth mediated intervention. The digital exercise intervention appeared to differentially benefit more sedentary participants, suggesting a target population that may profit most from the added mHealth support [14]. Limitations of this study included enrollment of a highly educated sample which was lacking in racial and ethnic diversity. This may influence the generalizability of the findings to the broader population with PD.

\section{DIGITAL THERAPEUTICS TO IMPROVE NON-MOTOR OUTCOMES}

Digital therapeutics need not be limited to approaches utilizing exercise or to those aimed to address motor aspects of the disease. Non-motor aspects of PD can also be addressed effectively via digital means. One of the most widely studied approaches in this realm is cognitive-behavioral therapy (CBT) delivered remotely [15]. Most recently, two relatively large RCTs in PD showed that remotely delivered CBT was superior to standard medical treatment in improving anxiety, depression, insomnia and quality of life $[16,17]$. These interventions were telephone-based or internet-based static educational modules. A digital CBT therapeutic in PD could offer automated, yet personalized and interactive treatment delivered at a time when the intervention is needed to address the symptoms of most concern. For example, in a singleblind RCT of 1711 community dwelling adults with probable DSM-5 insomnia disorder, digital CBT was delivered using web and/or mobile channels via the Sleepio programme and associated app (https:// www.bighealth.com/ sleepio and Sleepio App) and compared to sleep hygiene education [18]. The 6 -session program was fully automated and its underlying algorithms fed the delivery of information, support and advice tailored to the needs of the individual based on goals set by the participant at the outset of the program and sleep diary data provided by the patient daily. The program was highly interactive and content was presented by an animated virtual therapist. Treatment consisted of a behavioral component (i.e., sleep restriction, relaxation), a cognitive component (i.e., cognitive restructuring, mindfulness) and an educational component (i.e., sleep hygiene). Participants could view their progress, treatment strategies, sleep schedule and educational materials via the platform at their convenience. This digital CBT approach resulted in a small improvement in functional health and psychological well-being with a large improvement in sleep-related quality of life compared with sleep hygiene education after four weeks and 24 weeks. A large improvement in insomnia mediated these outcomes [19]. These results suggest the potential benefits of digitally applied CBT to improve sleep and present opportunities for application to PD. Similar digital therapeutic approaches to CBT have the potential be applied to reduce worry and anxiety (https://www.bighealth.com/daylight); however, studies are needed to determine efficacy in PD. If found to be efficacious in $\mathrm{PD}$, this digital approach to administering CBT interventions targeting various non-motor signs could be "prescribed" by physicians, allowing patients to access CBT at low cost in their own time and at their own pace.

\section{FUTURE PERSPECTIVES}

The potential benefits of digital therapeutics to patients, healthcare providers, and healthcare systems are substantial and far-reaching. Advances in artificial intelligence coupled with the central role of mobile technology in everyday life has expanded the 


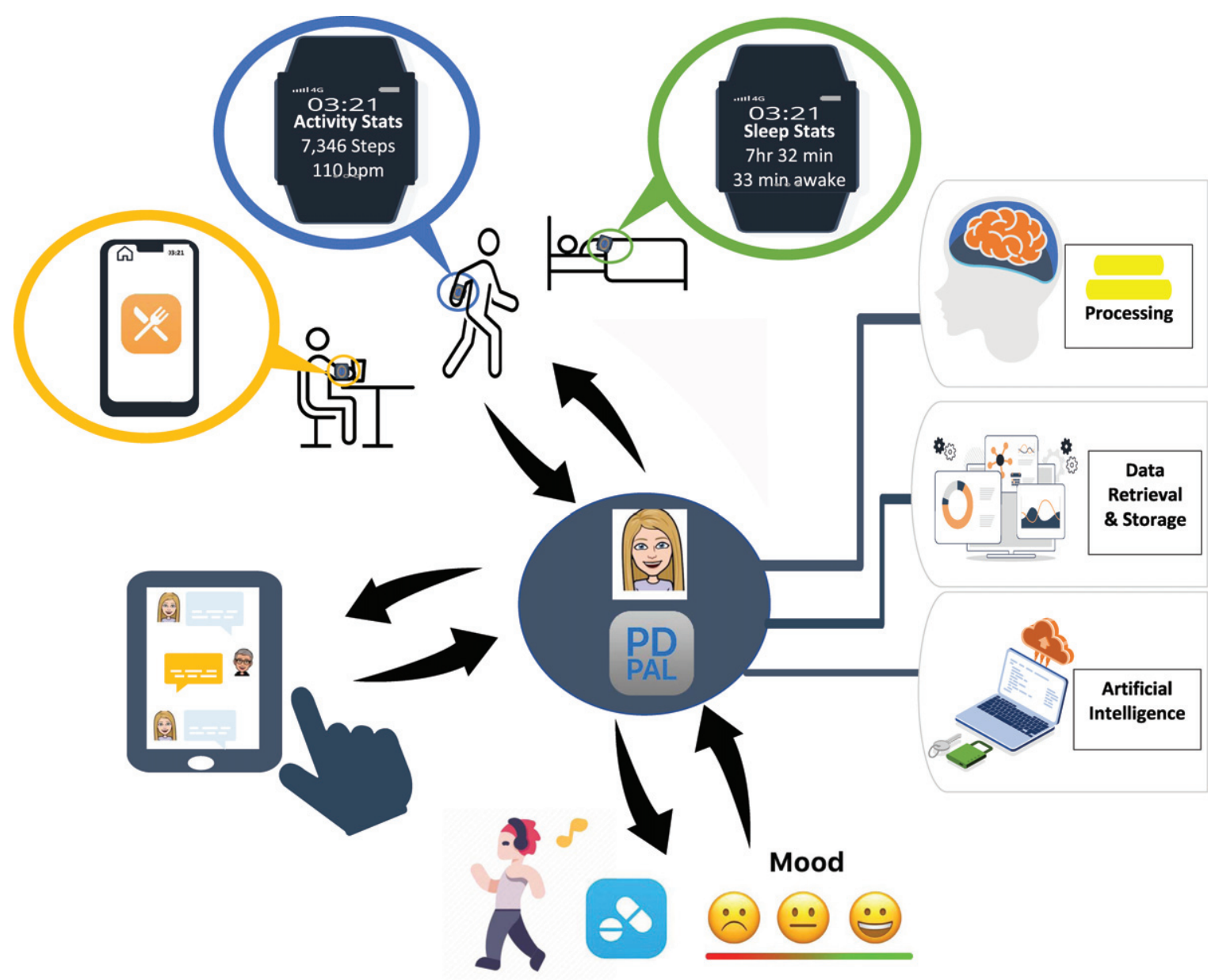

Fig. 2. Proposed Digital Therapeutic Platform Provides Centralized Hub for PD Management: "PD PAL" Artificially Intelligent Digital Therapeutic Platform provides a centralized hub (“one-stop shop”) for PD management by integrating data from physical activity, sleep, eating habits, motor (e.g., gait) and non-motor (e.g., mood) symptoms in relationship to medication intake to provide personalized digital interventions that address the areas of most concern or to achieve pre-determined goals.

application of digital therapeutics to healthcare. Clinical trials in digital therapeutics have increased more than fivefold over the past five years, and the market is expected to grow tenfold in the next three to five years [20]. This accelerated pace presents challenges related to how digital therapeutics need to be regulated, how healthcare providers and patients will respond and whether/how these technologies will be reimbursed by 3rd party payers/healthcare systems or provided through retail outlets. Much work remains to be done to advance digital therapeutics in PD, as studies in neurology constitute only $8 \%$ of digital therapeutics trials to date, with studies in rehabilitation lagging behind at less than $2 \%$ [20].

One vision for the future is to have a centralized "hub" which supports patients in managing a complex chronic condition, such as PD. Using an AIdriven approach, the platform (e.g., "PD PAL") could encompass a virtual coach that tailors care based on the needs of the individual (see Fig. 2). It would support all aspects of a patient's health by providing a whole-person approach through tracking and analyzing critical dimensions of health. It would sync with other data sources (e.g., activity trackers, sleep trackers, tremor) which could be passively collected during everyday living. It could prompt patients to complete periodic brief assessments of non-motor symptoms (e.g., anxiety, mood, cognition) and motor status (e.g., gait, balance) which could be integrated with data on when medications were taken. Relevant data would be shared and analyzed to enable coaching (e.g., goal setting, action planning, reminders, rewards) and personalized digital interventions (e.g., CBT for anxiety, depression or sleep or digital RAS for walking) addressing the most troubling symptoms at a given point in time. Ideally, the platform would 
integrate with various electronic medical record systems and summary data could be shared across the healthcare network to designated members of the interdisciplinary team. This approach would allow patients a "one-stop" centralized approach to management of all aspects of their PD.

Implementing a platform such as this will not be simple, as there are barriers that may limit uptake of digital therapeutics [21, 22]. Many digital therapeutics are accessed through smartphones. In a survey of adults $(\mathrm{N}=2,607)$ in the US aged $50+$ reflecting the demographics for American adults over 50, smartphone adoption was $86 \%$ for those 50-59 but drops to $62 \%$ for those 70 and older [23]. Although this suggests a relatively high prevalence of smartphone use among older adults, about $1 / 3$ of those over 70 do not use a smartphone, limiting access to many digital therapeutic options among this subgroup. A majority of African Americans (94\%) and Hispanic/Latinos $(91 \%)$ over age 50 use a smartphone; however, far fewer own other digital devices such as a fitness tracker, revealing a reduced adoption of digital devices beyond smartphones. We must acknowledge the digital divide and the fact that all digital offerings to date have primarily reached highly educated, white patients [24]. We must take care not to widen the gap in access as we move forward. There is great potential to reach those in underserved communities, but we are underachieving in this area. National policy changes are needed to bridge this digital divide along with local grassroots efforts to reach those patients who could benefit most.

In addition to these global barriers, there are some issues specific to PD that could impact adoption of digital therapeutics. Deficits in fine motor dexterity may necessitate increased button and icon sizes or use of a touch-pen. Training in the use of digital therapeutics may also be a challenge, as a recent study in PD revealed impairments in retention following fine motor skill training with a touchscreen swiping task [25]. Cognitive impairments may also interfere with integrating digital interventions in everyday life [26], and since many of the studies in PD examining the benefits of digital therapeutics exclude those with cognitive impairments it is not clear what cognitive capacity is needed to successfully use these technologies [6-14].

\section{CONCLUSION}

Digital therapeutics combines technology and evidence-based medicine to transform personalized patient care in an engaging and meaningful way. These approaches, although relatively early in their development, have great potential to improve access to evidence-based interventions to address unmet and widespread needs give patients with PD greater control over their treatment and enable access to the desired intervention along with progress updates at a convenient and time and place. Though promising, much greater advances are needed in PD to develop and integrate digital therapeutic options into the healthcare ecosystem. Importantly, engagement from all stakeholders (e.g., patients, care partners, healthcare providers, industry leaders, software engineers) is necessary to ensure a feasible, realistic, evidencebased solutions are generated.

\section{ACKNOWLEDGMENTS}

We would like to acknowledge funding from the National Institute of Child Health and Human Development (NICHD) \#1R01HD092444-01A1.

\section{CONFLICT OF INTEREST}

The authors have no conflict of interest to report.

\section{REFERENCES}

[1] Rajan R, Brennan L, Bloem BR, Dahodwala N, Gardner J, Goldman JG, Grimes DA, Iansek R, Kovács N, McGinley J, Parashos SA, Piemonte MEP, Eggers C (2020) Integrated care in Parkinson's disease: A systematic review and metaanalysis. Mov Disord 35, 1509-1531.

[2] Coravos A, Goldsack JC, Karlin DR, Nebeker C, Perakslis E, Zimmerman N, Erb MK (2019) Digital medicine: A primer on measurement. Digit Biomark 3, 31-71.

[3] https://dtxalliance.org/2019/11/11/digital-health-digitalmedicine-digital-therapeutics-dtx-whats-the-difference/

[4] Artusi CA, Imbalzano G, Sturchio A, Pilotto A, Montanaro E, Padovani A, Lopiano L, Maetzler W, Espay AJ (2020) Implementation of mobile health technologies in clinical trials of movement disorders: Underutilized potential. $\mathrm{Neu}$ rotherapeutics 17, 1736-1746.

[5] Sullivan AN, Lachman ME (2017) Behavior change with fitness technology in sedentary adults: A review of the evidence for increasing physical activity. Front Public Health 4, 289.

[6] Ellis T, Latham NK, DeAngelis TR, Thomas CA, SaintHilaire M, Bickmore TW (2013) Feasibility of a virtual exercise coach to promote walking in community-dwelling persons with Parkinson disease. Am J Phys Med Rehabil 92, $472-481$.

[7] Laranjo L, Dunn AG, Tong HL, Kocaballi AB, Chen J, Bashir R, Surian D, Gallego B, Magrabi F, Lau AYS, Coiera $\mathrm{E}$ (2018) Conversational agents in healthcare: A systematic review. JAMIA 25, 1248-1258.

[8] Maher CA, Davis CR, Curtis RG, Short CE, Murphy KJ (2020) A physical activity and diet program delivered by 
artificially intelligent virtual health coach: Proof-of-concept study. JMIR Mhealth Uhealth 8, e17558.

[9] Leuk JSP, Low LLN, Teo WP (2020) An overview of acoustic-based interventions to improve motor symptoms in Parkinson's disease. Front Aging Neurosci 12, 243.

[10] Ghai S, Ghai I, Schmitz G, Effenberg AO (2018) Effect of rhythmic auditory cueing on parkinsonian gait: A systematic review and meta-analysis. Sci Rep 8, 506.

[10] Hutchinson K, Sloutsky R, Collimore A, Adams B, Harris B, Ellis TD, Awad LN (2020) A music-based digital therapeutic: Proof-of-concept automation of a progressive and individualized rhythm-based walking training program after stroke. Neurorehabil Neural Repair 34, 986-996.

[12] Thomas Craig KJ, Morgan LC, Chen CH, Michie S, Fusco N, Snowdon JL, Scheufele E, Gagliardi T, Sill S (2020) Systematic review of context-aware digital behavior change interventions to improve health. Transl Behav Med, doi: 10.1093/tbm/ibaa099

[13] Michie S, Yardley L, West R, Patrick K, Greaves F (2013) Developing and evaluating digital interventions to promote behavior change in health and health care: Recommendations resulting from an international workshop. $J$ Med Internet Res 19, e232.

[14] Ellis TD, Cavanaugh JT, DeAngelis T, Hendron K, Thomas CA, Saint-Hilaire M, Pencina K, Latham NK (2019) Comparative effectiveness of mHealth-supported exercise compared with exercise alone for people with Parkinson disease: Randomized controlled pilot study. Phys Ther $\mathbf{9 9}$, 203-216.

[15] Luik AI, Kyle SD, Espie CA (2017) Digital cognitive behavioral therapy (dCBT) for insomnia: A state-of-the-science review. Curr Sleep Med Rep 3, 48-56.

[16] Dobkin RD, Mann SL, Gara MA, Interian A, Rodriguez KM, Menza M (2020) Telephone-based cognitive behavioral therapy for depression in Parkinson disease: A randomized controlled trial. Neurology 94, e1764-e1773.

[17] Kraepelien M, Schibbye R, Månsson K, Sundström C, Riggare S, Andersson G, Lindefors N, Svenningsson P, Kaldo V (2020) Individually tailored internet-based cognitivebehavioral therapy for daily functioning in patients with Parkinson's disease: A randomized controlled trial. $J$ Parkinsons Dis 10, 653-664.

[18] Espie CA, Luik AI, Cape J, Drake CL, Siriwardena AN, Ong JC, Gordon C, Bostock S, Hames P, Nisbet M, Sheaves B, G Foster R, Freeman D, Costa-Font J, Emsley R, Kyle SD (2016) Digital cognitive behavioural therapy for Insomnia versus sleep hygiene education: The impact of improved sleep on functional health, quality of life and psychological well-being. Study protocol for a randomised controlled trial. Trials 17, 257.

[19] Espie CA, Emsley R, Kyle SD, Gordon C, Drake CL, Siriwardena AN, Cape J, Ong JC, Sheaves B, Foster R, Freeman D, Costa-Font J, Marsden A, Luik AI (2019) Effect of digital cognitive behavioral therapy for insomnia on health, psychological well-being, and sleep-related quality of life: A randomized clinical trial. JAMA Psychiatry 76, 21-30.

[20] https://www.evidera.com/digital-therapeutics-past-trendsand-future-prospects/

[21] Espay AJ, Hausdorff JM, Sánchez-Ferro Á, Klucken J, Merola A, Bonato P, Paul SS, Horak FB, Vizcarra JA, Mestre TA, Reilmann R, Nieuwboer A, Dorsey ER, Rochester L, Bloem BR, Maetzler W (2019) A roadmap for implementation of patient-centered digital outcome measures in Parkinson's disease obtained using mobile health technologies. Mov Disord 34, 657-663.

[22] Dorsey ER, Glidden AM, Holloway MR, Birbeck GL, Schwamm LH (2018) Teleneurology and mobile technologies: The future of neurological care. Nat Rev Neurol 14, 285-297.

[23] Nelson Kakulla B (2020) 2020 Tech Trends of the 50+. AARP Research, Washington, DC. https://doi.org/ 10.26419/res.00329.001

[24] Dorsey ER, Achey MA, Beck CA, Beran DB, Biglan KM, Boyd CM, Schmidt PN, Simone R, Willis AW, Galifianakis NB, Katz M, Tanner CM, Dodenhoff K, Ziman N, Aldred J, Carter J, Jimenez-Shahed J, Hunter C, Spindler M, Mari Z, Morgan JC, McLane D, Hickey P, Gauger L, Richard IH, Bull MT, Mejia NI, Bwala G, Nance M, Shih L, Anderson L, Singer C, Zadikoff C, Okon N, Feigin A, Ayan J, Vaughan C, Pahwa R, Cooper J, Webb S, Dhall R, Hassan A, Weis D, DeMello S, Riggare SS, Wicks P, Smith J, Keenan HT, Korn R, Schwarz H, Sharma S, Stevenson EA, Zhu W (2016) National randomized controlled trial of virtual house calls for people with Parkinson's disease: Interest and barriers. Telemed J E Health 22, 590-598.

[25] Nackaerts E, Ginis P, Heremans E, Swinnen SP, Vandenberghe W, Nieuwboer A (2020) Retention of touchscreen skills is compromised in Parkinson's disease. Behav Brain Res 378, 112265.

[26] Bartels SL, van Knippenberg RJM, Malinowsky C, Verhey FRJ, de Vugt ME (2020) Smartphone-based experience sampling in people with mild cognitive impairment: Feasibility and usability study. JMIR Aging 3, e19852. 